UVX 2008 (2009) 29-34

(C) EDP Sciences, 2009

DOI: $10.1051 / \mathrm{uvx} / 2009006$

\title{
Amplificateurs paramétriques optiques pour la physique attoseconde
}

\author{
E. Cormier \\ CELIA, Université Bordeaux 1-CNRS-CEA, 351 cours de la Libération, 33405 Talence, \\ France
}

\begin{abstract}
Résumé. Dans cet article, nous discutons le potentiel des systèmes lasers basés sur la technique d'amplification paramétrique optique pour la production d'impulsions attosecondes par génération d'harmoniques d'ordre élevée. Nous montrons à travers plusieurs exemples que cette technologie a atteint la maturité suffisante pour répondre aux contraintes fortes exigées par la production d'impulsions attosecondes et plus particulièrement les impulsions attosecondes uniques.
\end{abstract}

\section{INTRODUCTION}

Depuis le début des années 2000, la physique à l'échelle attoseconde a connu un essor considérable lié à la possibilité de générer des impulsions attosecondes à partir des sources lasers développées dans les laboratoires. En effet, il a été démontré que la technique de génération d'harmoniques d'ordre élevé développée à partir des années 1990 permettait de produire des trains d'impulsions attosecondes dans le domaine XUV. Cependant, l'utilisation effective d'impulsions attosecondes pour résoudre la dynamique des phénomènes ultra-rapides en physico-chimie, en physique du solide et des plasmas nécessite la production d'impulsions attosecondes uniques. Ce dernier critère impose de disposer de sources lasers ultracourtes dont la durée se limite à quelques cycles optiques. Une des techniques permettant de produire de telles impulsions est basée sur l'amplification paramétrique optique (OPA) dans des milieux non-linéaires. Nous présenterons donc les différentes architectures menant à la production d'impulsions ultracourtes, leurs avantages, inconvénients et limitations au travers de systèmes actuels. Enfin, nous discuterons la stabilisation de la phase de la porteuse (CEP) dans ce contexte d'amplification.

\section{PRODUCTION D'IMPULSIONS ATTOSECONDES PAR GÉNÉRATION D'HARMONIQUES D'ORDRE ÉLEVÉ}

On sait depuis de nombreuses années que la production d'impulsions femtosecondes est possible dans le domaine infrarouge (photon de quelques eV) et nécessite des spectres dont l'énergie s'étale sur quelques dixièmes d'eV. En revanche, d'après la relation d'incertitude entre le temps et l'énergie, produire des pulses électromagnétiques dans le régime attoseconde requiert une bande spectrale de plusieurs dizaines d'eV. Une telle bande d'énergie couvre donc nécessairement le domaine VUVXUV (photons de plusieurs dizaines d'eV) des faibles longueurs d'ondes. La génération d'harmoniques d'ordre élevé est une technique fournissant un spectre XUV extrêmement large pouvant potentiellement supporter des impulsions attosecondes. En effet, le spectre produit par génération d'harmoniques doit posséder des propriétés particulières pour être compatible avec de véritables impulsions attosecondes. La possibilité de produire des impulsions attosecondes grâce á la génération d'harmoniques d'ordre élevé à été proposée il y a plus de 10 ans [1]. Le phénomène de génération d'harmoniques est observé lorsque l'on focalise un faisceau très intense (environ $10^{14} \mathrm{~W} / \mathrm{cm}^{2}$ ) sur une cible gazeuse [2]. Le spectre d'émission (voir figure 1) s'étalant dans l'UV se présente sous la forme d'un peigne de fréquence limité par une fréquence haute de coupure (faibles longueurs d'ondes). Chaque fréquence correspond à une harmonique impaire du laser. Dans le domaine temporel, l'intensité du faisceau d'harmoniques s'écrit 

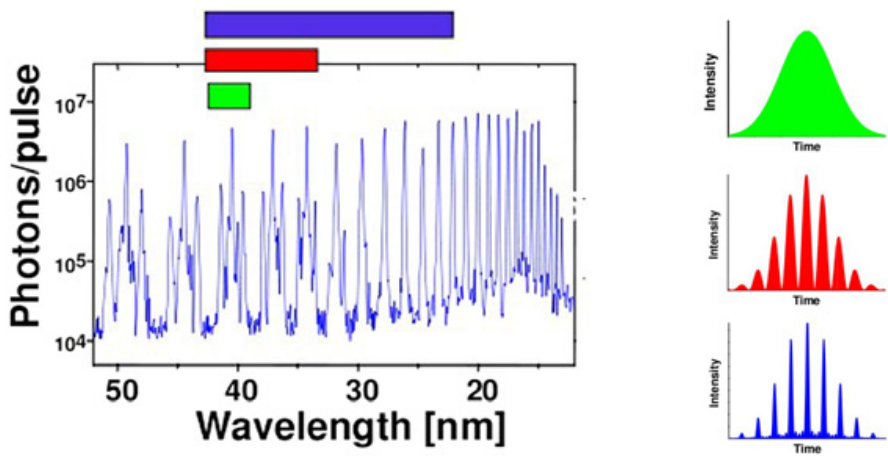

Figure 1. Spectre UVX obtenu par génération d'harmoniques d'ordre élevé (à gauche). Dépendence temporelle correspondante en fonction du nombre d'harmoniques sélectionnées (à droite).

donc comme la somme cohérente:

$$
I(t)=\left|\sum_{N} A_{q}(t) \exp \left(-i \omega_{q} t+i \phi_{q}\right)\right|^{2}
$$

où $A_{q}(t)$ est l'amplitude temporelle de l'harmonique $q$ et $\phi_{q}$ sa phase. Il a été démontré que chaque fréquence harmonique $q$ possède une relation de phase fixe avec les 2 fréquences voisines. C'est pour cette raison que l'on observe dans le domaine temporel une succession d'impulsions attosecondes de durée $\tau \approx \frac{T_{0}}{2 N}$ où $T_{0}$ est la période du laser et $\mathrm{N}$ le nombre d'harmoniques contribuant effectivement au signal UVX. On voit donc, comme illustré sur la figure 1, que plus le spectre d'harmoniques sélectionnées est large, plus les impulsions attosecondes du train sont courtes. C'est l'équivalent dans le domaine UVX du signal émergeant d'un oscillateur à blocage de modes infrarouge.

Le nombre d'impulsions attosecondes contenues dans le train dépend directement de la durée du pulse IR. Par exemple, pour une impulsion IR de $30 \mathrm{fs}$, le train contient plusieurs dizaines d'impulsions attosecondes ce qui limite le caractère ultracourt du rayonnement. En effet, il serait tout à fait intéressant de disposer d'une impulsion attoseconde unique permettant de bénéficier réellement de la brièveté de la source pour sonder la matière. En fait, générer une impulsion attoseconde quasiment isolée est possible en utilisant un faisceau de pompe dont la durée les impulsions n'excèdent pas 2 ou 3 cycles optiques. Dans ce cas, seul le cycle central où l'amplitude du champ est maximale sera générateur du rayonnement.

Cependant, l'utilisation d'impulsions de quelques cycles optiques apporte une contrainte suplémentaire liée à la phase relative de l'oscillation du champ par rapport à l'enveloppe de l'impulsion aussi appelée CEP (pour Carrier Envelop Phase). Suivant la valeur de la CEP, l'oscillation du champ électrique sera soit symétrique (sin) soit antisymétrique (cos) soit dans une configuration intermédiaire de sorte que la génération d'harmoniques produira une ou deux impulsions attosecondes. Le contrôle de la production d'impulsions attosecondes isolées nécessite donc la stabilisation et le contrôle de la CEP.

La production d'impulsions attosecondes par génération d'harmoniques d'ordre élevé impose donc de disposer d'une source primaire dont l'intensité atteint le régime de champ fort avec en plus, dans le cas d'impulsions attosecondes uniques, une durée d'impulsion limitée à quelques cycles optiques et dont la phase est stabilisée. La liste des sources actuelles répondant à ces critères sont données dans la tableau 1. On voit donc clairement que les sources basées sur l'amplification paramétrique optique constituent une alternative très prometteuse aux techniques de postcompression puisqu'elles allient impulsions ultra-courtes de très forte énergie et accordabilité. 
Tableau 1. Comparatif des sources primaires répondant aux critères nécessaires à la production d'impulsions attosecondes.

\begin{tabular}{l|c|c|c}
\hline & $\begin{array}{c}\text { Amplificateur } \\
\text { Ti:saphir }\end{array}$ & $\begin{array}{c}\text { Post-compression } \\
\text { (capillaire/filament) }\end{array}$ & OPA/NOPA/OPCPA \\
\hline$\lambda_{s}[\mathrm{~nm}]$ & 800 & 800 & $600-3000$ \\
$\mathrm{E}[\mathrm{mJ}]$ & $1-400$ & $0.5-3$ & $0.01-100$ \\
$\Delta \lambda[\mathrm{nm}]$ & 30 & 200 & octave \\
$\Delta \tau[\mathrm{fs}]$ & 30 & $4-12$ & $4-15$ \\
\hline
\end{tabular}

\section{L'AMPLIFICATION PARAMÉTRIQUE OPTIQUE}

La technique alternative qui nous intéresse particulièrement ici pour obtenir des impulsions ultracourtes est l'amplification paramétrique optique (OPA) proposée dans le début des années 60 [3,4]. Cette technique d'amplification a été initialement utilisée dans une cavité pour réaliser ce que l'on appelle un oscillateur paramétrique optique (OPO) [5,6]. Les propriétés du processus paramétrique optique utilisé comme moyen d'amplification optique permettent d'une part, de réduire significativement la durée des impulsions femtosecondes et, d'autre part de pouvoir ajuster la longueur d'onde d'émission de manière relativement simple [7,8]. Cette technique a été rapidement limitée par les seuils de dommage des éléments optiques du système. Ainsi, en 1992, Dubietis et al. [9] ont réalisé le premier OPCPA qui allie les propriétés de l'amplification paramétrique optique (OPA) et le concept d'amplification à dérive de fréquence (CPA). Cette approche permet donc la génération d'impulsions potentiellement très énergétiques afin d'atteindre des puissances crêtes collosales au niveau du PW[10] avec des systèmes de pompe "table top". L'amplification d'impulsions ultra-courtes par le principe OPA doit son succés à la réalisation de nouveaux cristaux à base de borates $(\mathrm{BBO}, \mathrm{LBO}, \ldots)$. La recherche et le développement dans le domaine de l'OPA/OPCPA est très active et d'importants résultats apparaissent dans la littérature à une fréquence élevée [11,12].
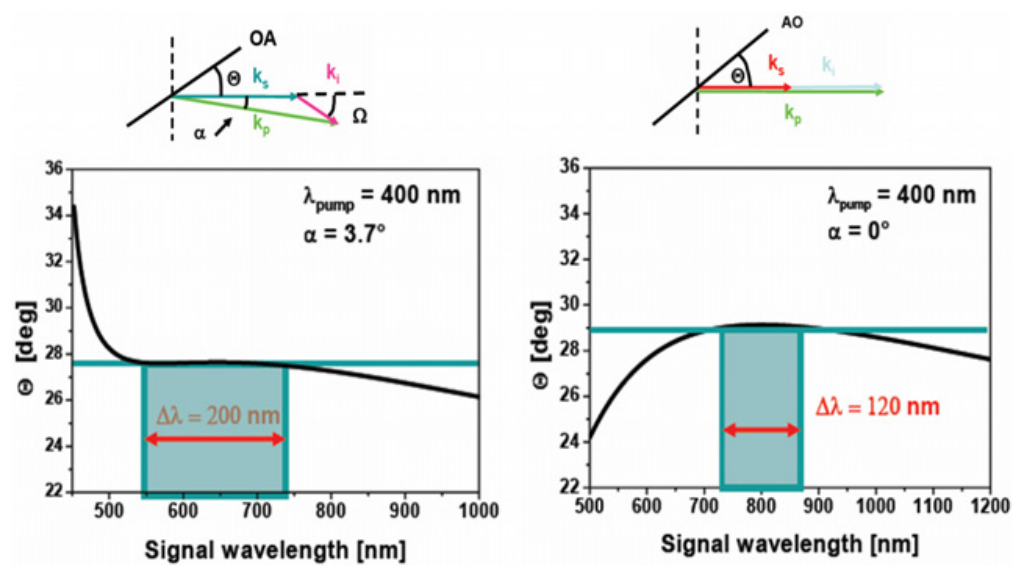

Figure 2. Courbes d'accord de phase dans un cristal de BBO de type I dans la configuration NOPA (non-colinéaire) à gauche et OPA (colinéraire) à droite pour un faisceau de pompe à $400 \mathrm{~nm}$.

L'amplification paramétrique optique est un phénomène d'optique non linéaire du deuxième ordre qui permet d'amplifier un faisceau signal de pulsation $\omega_{s}$ avec un faisceau de pompe de pulsation $\omega_{p}$ $\left(>\omega_{s}\right)$. Afin de vérifier la conservation de l'énergie $\left(\hbar \omega_{p}=\hbar \omega_{s}+\hbar \omega_{i}\right)$, un troisième faisceau, appelé faisceau complémentaire (ou idler), de pulsation $\omega_{i}=\omega_{p}-\omega_{s}$ est émis. L'énorme avantage de ce schéma d'amplification réside dans le fait qu'il est possible de choisir assez librement les longueurs d'ondes de la pompe et du signal ce qui rend la technique largement accordable. Ce processus conserve 
d'autre part l'impulsion de telle sorte que $\hbar \mathbf{k}_{\mathbf{p}}=\hbar \mathbf{k}_{\mathbf{s}}+\hbar \mathbf{k}_{\mathbf{i}}$. Cette loi détermine en fait les conditions d'accord de phase. En effet, le processus d'amplification n'est efficace que si les ondes se propagent dans le cristal non-linéaire à la même vitesse et donc en phase. Il est possible de jouer sur plusieurs paramètres pour obtenir l'accord de phase à la longueur d'onde souhaitée. Ces paramètres sont: la longueur d'onde du faisceau de pompe, le type de cristal (indices), l'angle du signal par rapport à l'axe optique du cristal, la température, l'angle entre le faisceau signal et le faisceau de pompe (géométrie non-collinéaire). On distinguera donc deux géometrie dans la réalisation d'un amplificateur: la géométrie collinéaire (OPA) et la géométrie non-collinéaire (NOPA) qui sont schématisées dans la figure 2. Par exemple, les courbes d'accord de phase de la figure 2 montrent clairement que pour une pompe à $400 \mathrm{~nm}$ et un angle du cristal donné, la bande spectrale amplifiable simultanément est extrèmement large. Elle est d'environ $100 \mathrm{~nm}$ en configuration OPA et de $200 \mathrm{~nm}$ en configuration NOPA. Le NOPA est donc préférable pour l'amplification d'impulsions ultracourtes. L'amplification paramétrique optique possède d'autre part des propriétés très intéressantes. Le processus étant instantanée, il n'y a pas de dépôt d'énergie dans le matériau (hormis l'absorption parasite du matériau) et donc pas d'effets thermiques. L'OPA est donc très bien adaptée à la très forte puissance moyenne. Le gain paramétrique peut atteindre $10^{4}$ à $10^{6}$ par passage ce qui limite le nombre d'étage d'amplification. Enfin, la qualité du faisceau amplifiée peut être excellente si celle du faisceau de pompe l'est aussi.

\section{LES SYSTÈMES PARAMÉTRIQUES ACTUELS}

Le potentiel de l'amplification paramétrique optique pour la production d'impulsions ultracourtes (quelques cycles optiques) nécessaire à la génération d'impulsions attosecondes uniques est donc clair. C'est pourquoi de nombreux groupes ont exploré cette technique depuis une dizaine d'année et ont obtenu des résultats très intéressants. On donne dans le tableau 2 une liste de systèmes réalisés récemment et mettant en oeuvre les différentes géométries possibles. Les paramètres de chaque système sont détaillés pour information. Afin de mieux appréhender le potentiel, les contraintes et les solutions proposées, nous présentons ici une sélection de systèmes basés sur l'amplification paramétrique optique. L'architecture des systèmes est résumée dans la figure 3. D'une manière générale, la relative facilité de production des impulsions ultracourtes repousse les contraintes sur la génération du signal et sur le système de pompage dont la qualité spatiale et temporelle est primordiale.

Tableau 2. Propriétés des systèmes paramétriques ultra-courts récents.

\begin{tabular}{ccccc|cc|ccc|c}
\hline & \multicolumn{3}{c}{ Pompage } & \multicolumn{4}{c}{ Amplificateur } & \multicolumn{3}{c}{ Sortie } \\
\hline Type & $\begin{array}{c}\lambda_{p} \\
{[\mathrm{~nm}]}\end{array}$ & $\begin{array}{c}E_{p} \\
{[\mathrm{~mJ}]}\end{array}$ & $\begin{array}{c}\tau \\
{[\mathrm{ps}]}\end{array}$ & $\begin{array}{c}\nu \\
{[\mathrm{Hz}]}\end{array}$ & $\begin{array}{c}\text { Config. } \\
{[\mathrm{mm}]}\end{array}$ & $\begin{array}{c}\text { Cristal } \\
{[\mathrm{nm}]}\end{array}$ & $\begin{array}{c}\lambda_{s} \\
{[\mathrm{fs}]}\end{array}$ & $\begin{array}{c}\tau \\
{[\mathrm{mJ}]}\end{array}$ & $E_{s}$ & Réf. \\
\hline Ti:S & 395 & 2.5 & 0.1 & 10 & NOPA & BBO (2) & 8 & 0.25 & {$[13]$} \\
Nd:Yag & 532 & 750 & 100 & 10 & NOPCPA & BBO (5) & 10 & 90 & {$[14]$} \\
Ti:S & 400 & $15+4$ & 100 & 1000 & NOPCPA & BBO (5) & 5.5 & 2.7 & {$[15]$} \\
Nd:Yag & 532 & $290+980$ & 9500 & 10 & NOPCPA & BBO(16) & 19 & 122 & {$[16]$} \\
Nd:Yag & 532 & 170 & 60 & 30 & NOPCPA & BBO(5.5) & 850 & 7.6 & 15.5 & {$[17]$} \\
Ti:S & 800 & 0.08 & 0.15 & 1000 & OPA & BBO(3) & 1600 & 8.5 & 0.004 & {$[18]$} \\
Ti:S & 790 & 10 & 0.06 & 10 & OPA & BBO(3) & 1600 & 17 & 1.2 & {$[19]$} \\
Nd:YLF & 1053 & 2 & 30 & 1000 & OPCPA & PPLT(3) & 2100 & 20 & & {$[20]$} \\
Nd:Yag & 532 & 160 & 60 & 30 & NOPA & BBO(5.5) & 850 & 7.6 & 15.5 & {$[21]$} \\
Ti:S & 390 & 0.01 & 0.18 & 1000 & NOPA & BBO (1) & 630 & 7.2 & 0.002 & {$[22]$} \\
Ti:S & 400 & 0.11 & & 1000 & OPCPA & LBO (8) & 910 & 15 & 0.01 & {$[23]$} \\
Nd:YAG & 532 & 50 & 60 & 20 & OPCPA & BBO (4) & 850 & 10 & 8 & {$[24]$} \\
\hline
\end{tabular}

\subsection{NOPCPA haute énergie}

Le système développé par Witte et al. [21] est basé sur 2 sources synchronisées électroniquement. Le signal est issu d'un oscillateur large bande délivrant des impulsions de 6 fs. Le deuxième oscillateur asservi au premier injecte une serie de 2 amplificateurs Nd:YAG. La pompe est doublée pour produire 

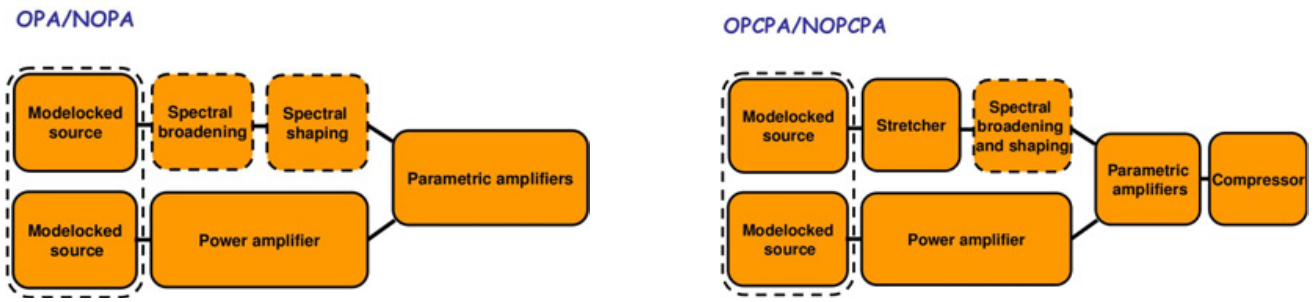

Figure 3. Architecture générale des systèmes paramétriques optiques (type OPA à gauche et OPCPA à droite). Les éléments en pointillés sont optionnels. Suivant les montages, les 2 sources lasers à blocage de modes nécessairement synchronisées sont parfois combinées en une seule source.

des impulsions de $60 \mathrm{ps}$ et $170 \mathrm{~mJ}$ à $532 \mathrm{~nm}$. Afin d'optimiser le recouvrement temporel entre la pompe et le signal, ce dernier est étiré dans un étireur dont la configuration est particulière. En effet, dans cette configuration, il est possible d'introduire un SLM (Spatial Light Modulator) dans le plan de fourier de l'étireur, où les fréquences sont dispersées spatialement, et ainsi procéder à un mise en forme spectrale. Le signal est ensuite amplifié par 2 étages de NOPA avant la recompression. Le système délivre finalement des impulsions de $7.6 \mathrm{fs}$ ( 2.7 cycles optiques) et $15.5 \mathrm{~mJ}$ soit $2 \mathrm{TW}$ à $30 \mathrm{~Hz}$. Un système similaire a été proposé par Tavella et al. [25]. L'originalité de cette architecture est double. D'une part, les auteurs s'affranchissent de la synchronisation des sources en n'utilisant qu'une seule source laser dont le spectre s'étale de $625 \mathrm{~nm}$ à $1070 \mathrm{~nm}$ couvrant ainsi les longueurs d'onde du signal et de la pompe. D'autre part, le couple étireur-compresseur est inversé. L'impulsion est initialement étirée par une paire de réseaux (dispersion négative) et recomprimée par un couple block de verre miroirs dispersifs occasionnant un minimum de pertes. Les impulsions ainsi obtenues sont de $8.5 \mathrm{fs}$ ( 3 cycles optiques) pour $80 \mathrm{~mJ}$ soit $10 \mathrm{TW}$ à $10 \mathrm{~Hz}$.

\subsection{NOPCPA et OPCPA ultra haute cadence}

Jusqu'à présent, les systèmes paramétriques utilisent des lasers de pompe à milieux massifs pour pouvoir disposer d'énergies conséquentes au détriment de la cadence. Depuis peu, la technologie à amplificateur fibré a atteint un niveau de développement qui permet aux FCPA (Fiber Chirped Pulse Amplifier) les plus récents d'atteindre une puissance de l'ordre du GW [26] (pour une durée de $800 \mathrm{fs}$ ) ou de $350 \mathrm{MW}$ [27] (pour une durée de $270 \mathrm{fs}$ ) à des cadences de $50 \mathrm{KHz}$ et $300 \mathrm{kHz}$ respectivement. Les impulsions délivrées par ces systèmes de très forte puissance moyenne ont une énergie suffisante pour pomper des étages d'amplificateurs paramétriques[28]. Hädrich et al. [29] ont ainsi démontré un OPCPA pompé par laser à fibre produisant des impulsions de moins de $30 \mathrm{fs}$ et $81 \mu \mathrm{J}$ à la cadence de $100 \mathrm{KHz}$. Nous travaillons actuellement sur un système similaire pompé par laser à fibre qui délivrera des impulsions de moins de 10 vfs (moins de 3 cycles optiques) stabilisé en phase, d'une énergie de quelques dizaines de $\mu \mathrm{J}$ à la cadence de $100 \mathrm{KHz}$. Dans ce système, le signal provient d'un oscillateur 6 fs stabilisé en phase. La largeur de bande de l'oscillateur est suffisante pour couvrir le spectre du signal autour de $800 \mathrm{~nm}$ et s'étendre jusqu'à $1030 \mathrm{~nm}$ pour injecter directement les étages d'amplificateurs à fibre dopées $\mathrm{Yb}$.

\subsection{OPA dans l'infra rouge proche}

Une autre approche consiste à utiliser comme source primaire la technologie titane-saphir largement disponible dans de nombreux laboratoires. Par exemple, Vozzi et al. [19] prélèvent 10\% du faisceau de la chaîne Ti:saphir pour générer le signal. Le reste sert directement de faisceau de pompe à $800 \mathrm{~nm}$. Les courbes d'accord de phase imposent donc, en configuration colinéaire, une amplification à la dégérescence autour de $1600 \mathrm{~nm}$. Le signal est généré de manière particulière. Un supercontinuum est 
d'abord créé par filamentation dans une cellule de gaz. Ce supercontinuum est ensuite recomprimé puis focalisé dans un cristal nonlinéaire. Le signal et l'idler (dégénéré dans le cas présent) sont alors créés par différence de fréquence. Cette technique possède l'énorme intérêt de générer un signal stabilisé en phase (CEP) à partir d'une source non-stabilisée. Le supercontinuum contient les radiations à 800 et $1600 \mathrm{~nm}$ dont les fluctuations de la CEP sont imposées par la source initiale. La troisième onde crée par différence de fréquence est complémentaire aux deux autres. Sa phase est donc la différence de phase des 2 signaux à 800 et $1600 \mathrm{~nm}$. Cette phase est constante quelque soit la valeur de la CEP du laser. Cette technique procure donc une auto-stabilisation de la CEP à moindre frais. Le dispositif ne possède ni étireur, ni compresseur, ni dispositif de doublage. Il émet des impulsions de 17 fs à $1,5 \mu \mathrm{m}$ (4 cycles optiques) avec une émergie de $1.2 \mathrm{~mJ}$.

\section{CONCLUSION}

La production de trains d'impulsions attosecondes par génération d'harmoniques d'ordre élevé dans un gaz et plus particulièrement l'obtention d'impulsions uniques nécessite une source primaire très intense, de seulement quelques cycles optiques et dont la phase (CEP) est stabilisée. Nous avons montré que la technique d'amplification paramétrique optique (OPA) et ses dérivées répond à tous les critères et se positionne comme une alternative très prometteuse aux techniques de postcompression d'impulsions issue de systèmes titane-saphir.

\section{Références}

[1] Antoine P. et al., PRL 77,(1996) 1234

[2] Ferray, M. et al., J. Phys. B 21, (1988) L31.

[3] S.A. Akhmanov and R.V. Khokhlov, Sov. Phys. JETP 16, (1963) 252.

[4] S.A. Akhmanov, et al., JETP Lett. 2, (1965) 191-193.

[5] N. M. Kroll et al., Phys. Rev. 127, (1962) 1207-1211.

[6] R. H. Kingston et al. Proc. IRE 50, (1962) 472.

[7] E. Riedle et al., Appl. Phys. B 71, (2000) 457-465.

[8] A. Baltuska et al., Opt. Lett. 27, (2002) 306- 308.

[9] A. Dubietis et al, Opt. Comm. 88, (1992) 437440.

[10] I. N. Ross et al., Opt. Commun. 144, (1997) 125133,.

[11] G. Cerullo and S. de Silvestri, Rev. Sci. Instrum. 74, (2003) 1-18.

[12] A. Dubietis et al., IEEE Journ. of Sel. Top. Quantum Electron. 12, (2006) 163172.

[13] Fülöp et al., New J. of Phys. 9, (2007) 438

[14] Tavella et al., Optics express, 14, (2006) 12822

[15] Adachi et al., Optics Express, 16, (2008) 14341

[16] Kiriyama et al., Optics Letters, 32, (2007) 2315

[17] Renault et al., Optics Letters 32, (2007) 2363

[18] Brida et al., Optics letters, 33, (2008) 741

[19] Vozzi et al., Optics letters, 32, (2007) 2957

[20] Fuji et al., Optics letters, 31, (2006) 1103

[21] Witte et al., Optics Express 14, (2006) 8168

[22] Cerullo et al., Optics Letters 23, (1998) 1283

[23] Tang et al., Optics Letters 33, (2008) 2386

[24] Ishii et al., Optics Letters 30, (2005) 567

[25] Tavella et al., Optics Letters 32, (2007) 2227

[26] Röser et al., Optics Letters 32, (2007) 3495

[27] Y. Zaouter et al., Optics Letters 33, (2008) 1527

[28] C. Aguergaray et al., Optics Express 15, (2007) 5699

[29] S. Hädrich et al., Optics Express 16, (2008) 19812 